\title{
Photon-photon interaction in axial channeling
}

\author{
J. Klenner, J. Augustin, A. Schäfer, and W. Greiner \\ Institut für Theoretische Physik, Universität Frankfurt am Main, Postfach 111 932, \\ D-60054 Frankfurt am Main, Germany
}

(Received 14 July 1993)

\begin{abstract}
We investigate the possibility that high-energy photons are channeled, when passing through an oriented single crystal, due to Delbrück scattering. For this purpose the exact electron propagator for the single-string model is constructed. Starting from a separation of variables, we solve the Dirac equation for a cylindrical electrostatic potential. The propagator for such external fields is constructed from solutions of the radial Dirac equation. This propagator is applied to a calculation of the $S$ matrix for Delbrück scattering. We specify the conditions under which photon channeling takes place. Unfortunately these conditions are only matched for a very small fraction of those photons being produced by channeled electrons.
\end{abstract}

PACS number(s): 12.20.Ds, 11.10.Qr, 03.65.Pm

\section{INTRODUCTION}

While QED is in general the best tested theory today, this is not true for some of its properties, most notably for the nonlinear electromagnetic interactions leading, e.g., to Delbrück scattering. In fact, the experimental results for Delbrück scattering only agree with the theoretical predictions for not too heavy atoms [1]. The reason for the discrepancies is most likely that present day calculations only take into account the contributions of lowest order in $Z$. Also, Delbrück scattering is experimentally very difficult to isolate as it interferes with Thomson scattering, Rayleigh scattering, and giant dipole scattering. Thus one has to conclude that the nonperturbative nonlinear effects of QED are presently hardly tested at all. There even has been much speculation about exotic nonperturbative effects in connection with the sharp electron-positron resonances observed at the Gesellschaft für Schwerionenforschung (Darmstadt); for a review see [2] and references therein. While none of these speculations should be taken too seriously they show how little is known about this sector of QED. It would therefore be very interesting to test such nonlinear and nonperturbative effects under different conditions, namely, for a photon under channeling conditions. With the latter we mean a photon moving very fast and very close to a string of atoms in an oriented single crystal. Then the virtual electron and positron coupling to the photon interact with the collective field of the whole row of atoms, an interaction that becomes highly nonperturbative for sufficiently large photon momentum. Due to this photon-photon interaction it might even happen that a fast photon under such conditions is channeled. We want to answer the question whether this actually can happen and if yes under which conditions. Far from being a purely academic question a favorable result could open most interesting perspectives for photon-photon physics. As argued in [3] one could make two electron beams collide under channeling conditions. If photons get bound to the channeling axes under such conditions this could dramatically increase the rates for photonphoton collisions.

\section{AXIAL CHANNELING AND THE SINGLE- STRING MODEL}

During the passage of high-energy particles through oriented single crystals a variety of nonperturbative phenomena occurs if the particles move parallel to a crystal axis or plane. These are called channeling phenomena. The basic mechanism is that a beam of charged incident particles nearly parallel to a symmetry axis or plane of the target crystal is scattered by collective interaction with whole axes or planes.

A lot of literature on this subject exists; e.g., [4-7], and references therein. We refer to them for the definition and determination of quantities like critical angles or channeling radiation spectra. We give only a brief heuristic motivation of the single-string model. If a particle moves parallel to an axis of atoms in a single crystal, the individual atomic potentials can be substituted by the averaged potential, neglecting the periodic oscillations in the direction of the motion. This leads to a cylindrical potential for every axis. The superposition of all these so-called string potentials gives the continuum model. For the string potential mainly two expressions are used. First the Lindhard potential, derived from a ThomasFermi-like atomic potential, and second the DoyleTurner potential, a sum of Gaussian curves fitted to relativistic calculations of electron scattering factors [8]:

$$
V_{\mathrm{DT}}(\rho)=Z_{1} e^{2} \frac{2 a_{0}}{d} \sum_{i=1}^{4} \frac{a_{i}}{B_{i}^{2}+R^{2}} \exp \left(\frac{-\rho^{2}}{B_{i}+R^{2}}\right) \text {. }
$$

Here $Z_{1}$ is the projectile's charge, $d$ the distance between two atoms of one row, $a_{0}$ the Bohr radius, $R^{2}$ describes the size of thermal vibration in the transverse plane, and the constants $a_{i}, B_{i}$ are tabulated for different targets [5]. (The Doyle-Turner potential for an electron channeling along the $\langle 110\rangle$ axis in a germanium target at room temperature is shown in Fig. 2.)

At this point we have to decide what effects should be investigated. For example, negative projectiles flying nearly parallel to the axes with low transverse energy can 


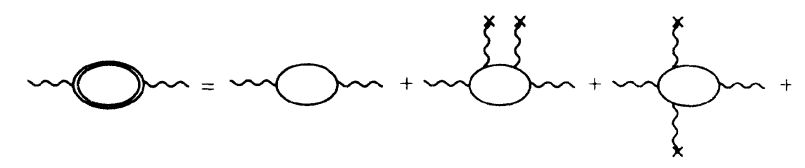

FIG. 1. Delbrück scattering.

be bound to one particular axis, this means string, because of the positive charges of the nuclei. Therefore it is intuitively permitted to neglect all other but the one string of interest, leading to the cylindrical single-string model, called SSM in the following.

On the other hand, positive projectiles with low transverse energy can be bound in the transverse plane between some strings. This channeling effect has recently been used to bend high-energy proton beams [9], but for these particles the SSM is not a good description of the crystal, because they are captured by several strings.

The situation for photons is unclear. So far nobody has studied the effects of photon-photon interactions in detail. However, if the bremsstrahlung photons emitted by channeled electrons were to be channeled too, this could be used to obtain high photon-photon luminosities [3]. This more practical motivation and the principal question of photon channeling induced us to investigate that possibility within the SSM.

The basic process for the scattering of photons by an external field, called Delbrück scattering, is shown in Fig. 1. The photon is scattered via the interaction of virtual electron positron pairs with the external field.

Since the interaction of the charged particles is maximal for maximal electric field it is permissible to calculate the cross section within the SSM and sum up all contributions of different strings. (See Fig. 2, the maximal electric field is at $\rho \approx 0.125 \AA$, a small quantity compared to the distances between two axes.)

For our calculation we take plane waves for the photons and the exact electron propagators for the fermions of the loop. This corresponds to lowest order in $\alpha$ and includes all orders in the external field.

The Doyle-Turner potential has a certain similarity to a cut-off Coulomb potential as shown in Fig. 2. Intuitively one would say that the description in terms of a Coulomb-like potential would give the same order of magnitude for the $S$ matrix of Delbrück scattering. The

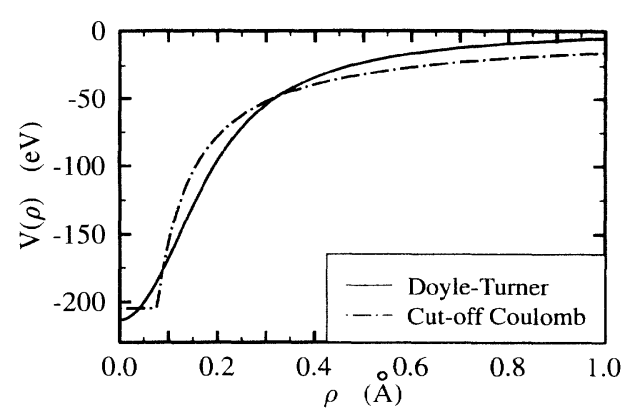

FIG. 2. Doyle-Turner potential and a cut-off Coulomb potential; see text. description in terms of a Coulomb-like potential has the advantage that the solutions of the Dirac equation are given analytically in terms of Bessel and Whittaker functions.

The calculation of matrix elements of the resolvent operator showed that the propagators in the two different descriptions are numerically similar [10]. For our problem it turned out that the Coulomb description is not very helpful, since Bessel and Whittaker functions are not easy to calculate numerically for the specific parameters and arguments needed. We therefore used the frame of the SSM with the above described Doyle-Turner potential for the $\langle 110\rangle$ axis in germanium at room temperature and calculated the propagators numerically.

\section{DIRAC EQUATION IN CYLINDRICAL COORDINATES}

To describe QED effects in the SSM we need to solve the Dirac equation for a cylindrical potential $A^{\mu}$ $=\left(A^{0}(\rho), 0\right)$. This is done according to the more general considerations in $[11,12]$.

With the electron's charge $q=-|e|$ the Dirac equation for an external field reads-we use $\hbar=c=1$ and the Dirac representation for the $\gamma$ matrices [13]:

$$
\left[\alpha \cdot(\mathbf{p}-q \mathbf{A})+\beta m_{e}+q A^{0}\right] \Psi(\mathbf{x}, t)=i \partial_{t} \Psi(\mathbf{x}, t) .
$$

For the SSM we have $q A^{0}(\rho)=V(\rho)$ and after multiplication with $\gamma^{0}$, (2) simplifies to

$$
\left[-i\left(\gamma^{1} \partial_{x}+\gamma^{2} \partial_{y}+\gamma^{3} \partial_{z}\right)+m_{e}+(V-E) \gamma^{0}\right] \Psi(\mathbf{x})=0,
$$

$\Psi(\mathbf{x}, t)=e^{-i E t} \Psi(\mathbf{x})$.

First we substitute the corresponding expression in $\partial_{\rho}, \partial_{\varphi}, \partial_{z}$ for the Cartesian operator $\boldsymbol{\gamma} \cdot \boldsymbol{\nabla}$. Our second step is to make a nonunitary transformation of the resulting equation. The operator of this transformation, namely,

$$
T=\sqrt{\rho} \exp \left(-\gamma^{1} \gamma^{2} \frac{\varphi}{2}\right)
$$

is not unitary owing to the factor $\sqrt{\rho}$, which is introduced for later convenience. (Note that the volume element in cylindrical coordinates read $\rho d \rho d \varphi d z$.) With

$$
\begin{aligned}
& \Psi \rightarrow \widetilde{\Psi}=T \Psi, \\
& \gamma^{0}(H-E) \rightarrow T \gamma^{0}(H-E) T^{-1},
\end{aligned}
$$

we are led to

$$
\left\{-i\left[\gamma^{1} \partial_{\rho}+\gamma^{2} \frac{1}{\rho} \partial_{\varphi}+\gamma^{3} \partial_{z}\right]+m_{e}+(V-E) \gamma^{0}\right\} \widetilde{\Psi}=0 .
$$

The next step is to write an equation with a once more transformed wave function $\Psi^{\prime}$ and a transformed operator, expressed as a sum of two operators $K_{1}$ and $K_{z}$, depending only on $\rho, \varphi$, and $z$, respectively:

$$
\left\{K_{\perp}+K_{z}\right\} \Psi^{\prime}=0,\left[K_{\perp}, K_{z}\right]=0 \text {. }
$$


Since we want to separate the variables we demand that the commutator of these two operators vanish.

For this new equation to be equivalent with (6) we make the ansatz

$$
\begin{aligned}
& K_{\perp}=\left\{-i\left[\gamma^{1} \partial_{\rho}+\gamma^{2} \frac{1}{\rho} \partial_{\varphi}\right]+(V-E) \gamma^{0}\right\} \Gamma, \\
& K_{z}=\left\{-i \gamma^{3} \partial_{z}+m_{e}\right\} \Gamma, \\
& \widetilde{\Psi} \rightarrow \Psi^{\prime}=\Gamma^{-1} \widetilde{\Psi},
\end{aligned}
$$

with a $4 \times 4$ matrix $\Gamma$. The vanishing commutator in (7) leads to several equations determining $\Gamma$, with the solution

$$
\Gamma=\gamma^{1} \gamma^{2} \gamma^{0} .
$$

In the representation (8) the operators $K_{\perp}$ and $K_{z}$ mix the upper and lower components of the spinor $\Psi^{\prime}$. This unpleasant feature is removed by the unitary transformation

$$
\begin{aligned}
& S=\frac{1}{\sqrt{2}}\left(1+i \gamma^{0} \gamma^{3}\right), \\
& S^{-1}=\frac{1}{\sqrt{2}}\left(1-i \gamma^{0} \gamma^{3}\right), \\
& \Psi^{\prime} \rightarrow \Psi_{n}=S^{-1} \Psi^{\prime}, \\
& \gamma^{\mu} \rightarrow S^{-1} \gamma^{\mu} S .
\end{aligned}
$$

The transformed operators are given by

$$
\begin{aligned}
& K_{\perp} \rightarrow K_{\perp}^{\prime}=-\left[\gamma^{2} \gamma^{3} \partial_{\rho}-\gamma^{1} \gamma^{3} \frac{1}{\rho} \partial_{\varphi}\right]+(V-E) \gamma^{1} \gamma^{2}, \\
& K_{z} \rightarrow K_{z}^{\prime}=\gamma^{5} \partial_{z}+m_{e} \gamma^{0} \gamma^{5} .
\end{aligned}
$$

Since the commutator of $K_{\perp}^{\prime}$ and $K_{z}^{\prime}$ vanishes too, we make the following ansatz:

$$
\left[K_{1}^{\prime}, K_{z}^{\prime}\right]=0 \Longrightarrow K_{\perp}^{\prime} \Psi_{n}=-i \varepsilon \Psi_{n} \text { and } K_{z}^{\prime} \Psi_{n}=+i \varepsilon \Psi_{n} \text {, }
$$

with a wave function $\Psi_{n}$, consisting of a plane wave in the $z$ direction, a phase factor containing the angular momentum $\mu$ (see below), and a spinor depending on $\rho$ :

$$
\Psi_{n}=e^{i p_{z}^{z}} e^{i \mu \varphi}\left[\begin{array}{l}
\mathbf{u}(\rho) \\
\mathbf{v}(\rho)
\end{array}\right] .
$$

After a short calculation this leads to the following relations:

$$
\mathbf{v}=\frac{-i \varepsilon}{i p_{z}+m_{e}} \mathbf{u}, \quad \varepsilon^{2}=p_{z}^{2}+m_{e}^{2},
$$

and the radial Dirac equation for a cylindrical electrostatic potential

$$
\left[\begin{array}{cc}
\frac{d}{d \rho}-\frac{\mu}{\rho} & -\left[E+s m_{\perp}-V(\rho)\right] \\
E-s m_{\perp}-V(\rho) & \frac{d}{d \rho}+\frac{\mu}{\rho}
\end{array}\right]\left[\begin{array}{l}
u_{1} \\
u_{2}
\end{array}\right]=0 .
$$

Here $s m_{\perp}=\varepsilon= \pm \sqrt{p_{z}^{2}+m_{e}^{2}}$ denotes the transverse mass $m_{\perp}$ multiplied by $s= \pm 1$. The meaning of this constant will be briefly discussed below. Before noting the wave function, we want to emphasize that this radial equation is essentially the same as for spherically symmetric potentials.

With $u$ being a solution to the radial equation (15) the solution $\Psi$ of the Dirac equation (3) is given by the following expression:

$$
\begin{aligned}
\Psi(\rho, \varphi, z, t)= & \frac{1}{\sqrt{2}} \frac{e^{i\left[p_{z} z-E t\right]}}{\sqrt{2 \pi}} \frac{e^{i \mu \varphi}}{\sqrt{2 \pi}} \exp \left(\gamma^{1} \gamma^{2} \frac{\varphi}{2}\right) \gamma^{1} \gamma^{2} \gamma^{0} \\
& \times \frac{1}{\sqrt{2}}\left(1+i \gamma^{0} \gamma^{3}\right) \frac{1}{\sqrt{\rho}}\left(\begin{array}{c}
\mathbf{u} \\
\frac{-i s m_{\perp}}{i p_{z}+m_{e}} \mathbf{u}
\end{array}\right)
\end{aligned}
$$

There are three constants appearing in the derivation of Eqs. (15) and (16), which we want to assign to physical quantities:

(i) $\Psi$ is obviously an eigenfunction of $J_{z}=-i \partial_{\varphi}$ $+(i / 2) \gamma^{1} \gamma^{2}$, the operator of total angular momentum in the $z$ direction, with $\mu$ as an eigenvalue. (Observe that $\left.\left[H, J_{z}\right]=0\right)$. For Dirac particles $\mu$ can take half integer values

$$
\mu= \pm \frac{1}{2}, \pm \frac{3}{2}, \pm \frac{5}{2}, \ldots \text {. }
$$

(ii) $m_{1}=+\sqrt{p_{z}^{2}+m_{e}^{2}}$ plays the role of the mass for the transverse motion. It is an interesting detail that in the radial equation (15) this mass is multiplied by the sign factor $s$.

(iii) The factor $s$ always occurring in the combination $s m_{\perp}$ has no direct physical interpretation, but in the nonrelativistic and in the ultrarelativistic limit $s= \pm 1$ gives the $z$ component of the electron's spin. $\Psi$ is the eigenfunction of the operator $\Sigma=\left(1 / m_{1}\right)\left(-i \partial_{z} \gamma^{5}+m_{e} \gamma^{3} \gamma^{5}\right)$, $[H, \Sigma]=0$, with eigenvalue $s$.

Finally we want to note that every solution is characterized by four quantum numbers, namely, the total energy $E$, the longitudinal momentum $p_{z}$, the total angular momentum in the $z$ direction $\mu$, and the "spin" variable $s$.

\section{EXACT ELECTRON PROPAGATOR}

Now we are able to calculate the exact electron propagator $S_{F}$ in a cylindrical external electrostatic field, as shown in Fig. 3. $S_{F}$ sums up the interaction of the electron with the external field to all orders.

Mathematically $S_{F}$ is given as the Green function of the Dirac operator including the external field [13],

$$
\left[\gamma^{\mu}\left(i \partial_{\mu}-q A_{\mu}\right)-m_{e}\right] S_{F}\left(x, x^{\prime}\right)=\delta^{4}\left(x-x^{\prime}\right),
$$

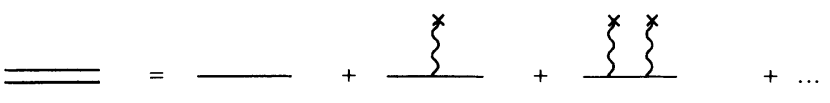

FIG. 3. Diagrammatic representation of $S_{F}$. 
and by a Fourier transform it is related to the resolvent or the Green function $G$ :

$$
i S_{F}\left(\mathbf{x}, \mathbf{x}^{\prime}, t-t^{\prime}\right) \gamma^{0}=\int_{C_{F}} \frac{e^{-i\left(t-t^{\prime}\right) \xi}}{2 \pi i} G\left(\mathbf{x}, \mathbf{x}^{\prime}, \zeta\right) d \zeta .
$$

The contour $C_{F}$ of integration in the plane of complex energy $\zeta$ determines the vacuum. For our purposes it is the usual Feynman contour as used for the free propagator. Formally the resolvent $G$ is given by a sum over products of eigenfunctions, with $\alpha$ representing all quantum numbers

$$
G\left(\mathbf{x}, \mathbf{x}^{\prime}, \zeta\right)=\oiint_{\alpha} \frac{\Psi_{\alpha}(\mathbf{x}) \Psi_{\alpha}^{\dagger}\left(\mathbf{x}^{\prime}\right)}{E_{\alpha}-\zeta}
$$

Following the idea of [14] for spherically symmetric potentials we take (20) seriously, working out the product $\Psi \Psi^{\dagger}$ and combining all terms depending on $\rho, \rho^{\prime}$ to four unknown functions $k_{i j}\left(\rho, \rho^{\prime}\right)$ :

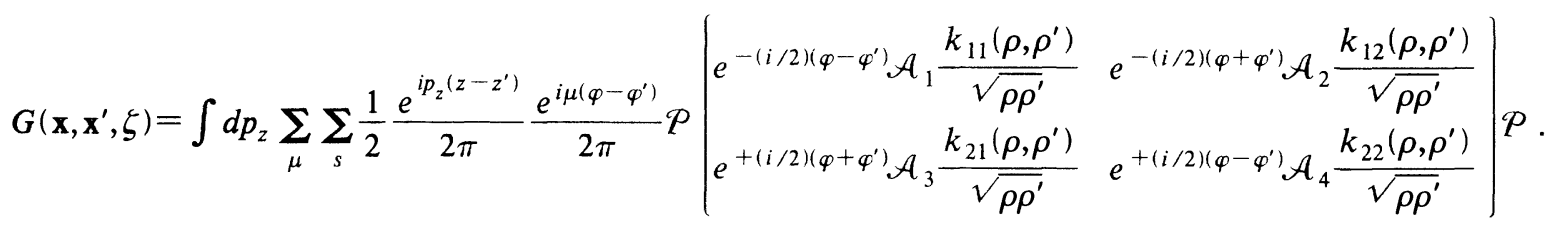

The $\mathcal{A}_{i}$ are $2 \times 2$ matrices given by

$$
\begin{aligned}
& \mathcal{A}_{1 / 4}=\left(\begin{array}{cc}
1 \pm \frac{m_{e}}{s m_{\perp}} & \frac{p_{z}}{s m_{\perp}} \\
\frac{p_{z}}{s m_{\perp}} & 1 \mp \frac{m_{e}}{s m_{\perp}}
\end{array}\right), \\
& \mathcal{A}_{2 / 3}= \pm\left(\begin{array}{cc}
i \frac{p_{z}}{s m_{\perp}} & i\left(1 \pm \frac{m_{e}}{s m_{\perp}}\right) \\
i\left(1 \mp \frac{m_{e}}{s m_{\perp}}\right) & i \frac{p_{z}}{s m_{\perp}}
\end{array}\right),
\end{aligned}
$$

and $\mathcal{P}$ denotes a $4 \times 4$ matrix just permuting the second and third component,

$$
P=\left(\begin{array}{llll}
1 & 0 & 0 & 0 \\
0 & 0 & 1 & 0 \\
0 & 1 & 0 & 0 \\
0 & 0 & 0 & 1
\end{array}\right)
$$

Putting this ansatz for $G$ into Eqs. (18) and (19) for $S_{F}$ gives the following equation for the unknown functions $k_{i j}$ :

$$
\left[\begin{array}{cc}
s m_{\perp}+V(\rho)-\xi & -\frac{d}{d \rho}-\frac{\mu}{\rho} \\
\frac{d}{d \rho}-\frac{\mu}{\rho} & -s m_{\perp}+V(\rho)-\zeta
\end{array}\right] \begin{aligned}
& \left(k_{i j}\left(\rho, \rho^{\prime}\right)\right) \\
& =\delta\left(\rho-\rho^{\prime}\right)
\end{aligned} .
$$

From [15] we know a solution to (24) in terms of solutions of the radial Dirac equation. If $\mathbf{v}$ and $\mathbf{w}$ denote solutions of (15) for complex energy $\zeta$, regular at the origin and infinity, respectively, one representation for the radial Green functions is given by

$k_{i j}\left(\rho, \rho^{\prime}\right)=\frac{\Theta\left(\rho^{\prime}-\rho\right)}{K(\zeta)} v_{i}(\rho) w_{j}\left(\rho^{\prime}\right) \frac{\Theta\left(\rho-\rho^{\prime}\right)}{K(\zeta)} w_{i}(\rho) v_{j}\left(\rho^{\prime}\right)$.
$K(\zeta)$ is the Wronskian of $\mathbf{v}$ and $\mathbf{w}$, and is independent of $\rho:$

$$
\begin{aligned}
& K(\zeta)=v_{2}(\rho, \zeta) w_{1}(\rho, \zeta)-v_{1}(\rho, \zeta) w_{2}(\rho, \zeta), \\
& 0<\rho<\infty .
\end{aligned}
$$

For practical calculations it should be noticed that the functions $k_{i j}$ for $-\mu,-s$ are simply related to that for $+\mu,+s$ :

$$
\begin{aligned}
& \left(\begin{array}{ll}
k_{11}(-\mu,-s) & k_{12}(-\mu,-s) \\
k_{21}(-\mu,-s) & k_{22}(-\mu,-s)
\end{array}\right) \\
& =\left(\begin{array}{ll}
+k_{22}(+\mu,+s) & -k_{21}(+\mu,+s) \\
-k_{12}(+\mu,+s) & +k_{11}(+\mu,+s)
\end{array}\right] .
\end{aligned}
$$

For the calculation of Delbrück scattering we need another property of $\mathbf{v}$ and $\mathbf{w}$. For $\rho \gg 1$ they asymptotically behave like

$$
\mathbf{v} \cong e^{-M \rho}, \quad \mathbf{w} \cong e^{+M \rho}, \quad M=\left(m_{1}^{2}-\xi^{2}\right)^{1 / 2},
$$

with an effective mass $M$ for the propagation in the radial direction. (We take the square root with non-negative real part.) With two new spinors,

$$
\widetilde{\mathbf{v}} \equiv e^{+M \rho} \mathbf{v}, \quad \widetilde{\mathbf{w}} \equiv e^{-M \rho} \mathbf{w},
$$

the radial Green function reads

$$
\begin{aligned}
k_{i j}\left(\rho, \rho^{\prime}\right)=e^{-M\left|\rho-\rho^{\prime}\right|} & \left(\frac{\Theta\left(\rho^{\prime}-\rho\right)}{K(\zeta)} \widetilde{v}_{i}(\rho) \widetilde{w}_{j}\left(\rho^{\prime}\right)\right. \\
& \left.+\frac{\Theta\left(\rho-\rho^{\prime}\right)}{K(\xi)} \widetilde{w}_{i}(\rho) \widetilde{v}_{j}\left(\rho^{\prime}\right)\right] .
\end{aligned}
$$

In this form the exponentially damped propagation for "massive particles" is shown explicitly.

At this point a serious problem occurs when using the Coulomb-like potential. Since $M \approx \omega / 2 \gg 1$ for our purposes, we need asymptotic expansions for the solutions of the radial Dirac equation, taking into account the exponential behavior. There exist formulas of this kind, but they do not work for radii $\rho \approx 1 \AA$ or bigger [10]. 


\section{DELBRÜCK SCATTERING}

This section is devoted to the calculation of $S$ matrix elements of Delbrück scattering in the SSM with the previously defined Doyle-Turner potential. From quantum field theory we know the $S$ matrix elements,

$$
\begin{aligned}
& S_{f i}=\int d^{4} x_{1} d^{4} x_{2}\left\langle f\left|:(\hat{\bar{\Psi}} A \hat{\Psi})\left(x_{1}\right)(\hat{\bar{\Psi}} A \hat{\Psi})\left(x_{2}\right):\right| i\right\rangle \\
&=e^{2} \int d^{4} x_{1} d^{4} x_{2} \operatorname{Tr}[ i S_{F}\left(x_{1}, x_{2}\right) A_{f}\left(x_{1}\right) \\
&\left.\times i S_{F}\left(x_{2}, x_{1}\right) A_{i}\left(x_{2}\right)\right],
\end{aligned}
$$

where the Dirac fields $\hat{\Psi}, \hat{\bar{\Psi}}$ are operators in the external field. The initial and final photon states $|i\rangle,|f\rangle$ are represented by plane waves, each one with two transverse polarization vectors

$$
\begin{aligned}
& \varepsilon_{i}^{(1)}=\omega_{i}^{-1}\left(0, k_{\|}, 0,-k_{\perp}\right), \\
& \varepsilon_{i}^{(2)}=(0,0,1,0),
\end{aligned}
$$

$$
\begin{aligned}
& \varepsilon_{f}(1)=\omega_{f}^{-1}\left(0, k_{\|} \cos \phi, k_{\|} \sin \phi,-k_{\perp}\right), \\
& \varepsilon_{f}^{(2)}(0,-\sin \phi, \cos \phi, 0) .
\end{aligned}
$$

$\omega_{i}=\omega_{f} \equiv \omega$ denotes the energy of incoming and outgoing photons. Since it is scattered off an external static field the energy is conserved. In addition to longitudinal component $k_{\|}$and therefore the absolute value $k_{1}$ of the transverse photon momentum are conserved too. The only possible effect of the scattering process is a rotation of the transverse photon momentum in the $k_{x}-k_{y}$ plane by an angle $\phi$.

The plane waves can be expanded in Bessel functions using

$$
e^{i x \cos \alpha}=\sum_{m=-\infty}^{+\infty} i^{m} e^{i m \alpha} J_{m}(x),
$$

and after some algebra the following equation for $S$ is derived:

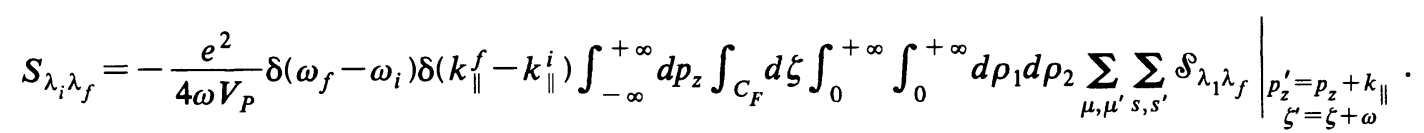

Primed and unprimed quantities refer to the propagators $S_{F}\left(x_{2}, x_{1}\right)$ and $S_{F}\left(x_{1}, x_{2}\right)$, respectively, and $V_{P}$ is the normalization volume for the electromagnetic field. The polarizations of initial and final photons are numbered by $\lambda_{i}, \lambda_{f}$. With the following abbreviations,

$$
\begin{aligned}
& J_{\mu^{\prime}-\mu( \pm 1)}=J_{\mu^{\prime}-\mu( \pm 1)}\left(k_{\perp} \rho_{1}\right), J_{\mu-\mu^{\prime}( \pm 1)}=J_{\mu-\mu^{\prime}( \pm 1)}\left(-k_{\perp} \rho_{2}\right), \\
& T_{1}=\frac{p_{z} p_{z}^{\prime}+s s^{\prime} m_{\perp} m_{\perp}^{\prime}}{s s^{\prime} m_{\perp} m_{\perp}^{\prime}+m_{e}^{2}}, T_{2}=\frac{p_{z}}{s m_{\perp}}+\frac{p_{z}^{\prime}}{s^{\prime} m_{\perp}^{\prime}}, T_{3}=\frac{p_{z} p_{z}^{\prime}+s s^{\prime} m_{\perp} m_{\perp}^{\prime}-m_{e}^{2}}{s s^{\prime} m_{\perp} m_{\perp}^{\prime}}, \\
& k_{i j}=k_{i j}\left(\rho_{1}, \rho_{2}\right), \quad k_{i j}^{\prime}=k_{i j}^{\prime}\left(\rho_{2}, \rho_{1}\right),
\end{aligned}
$$

the "reduced matrix elements" $\oint_{\lambda_{i} \lambda_{f}}$ are given by

$$
\begin{aligned}
& \mathcal{S}_{11}=e^{-i\left(\mu-\mu^{\prime}\right) \phi} \frac{i}{\omega^{2}}\left(\left\{k _ { \| } ^ { 2 } T _ { 1 } \left(J_{\mu^{\prime}-\mu+1} J_{\mu-\mu^{\prime}-1} e^{+2 i \phi} k_{11} k_{22}^{\prime}+J_{\mu^{\prime}-\mu+1} J_{\mu-\mu^{\prime}+1} k_{12} k_{12}^{\prime}\right.\right.\right. \\
& \left.+J_{\mu^{\prime}-\mu-1} J_{\mu-\mu^{\prime}+1} e^{-2 i \phi} k_{22} k_{11}^{\prime}+J_{\mu^{\prime}-\mu-1} J_{\mu-\mu^{\prime}-1} k_{21} k_{21}^{\prime}\right) \\
& +k_{\|} k_{1} T_{2}\left[J_{\mu^{\prime}-\mu} J_{\mu-\mu^{\prime}+1} e^{-i \phi}\left(k_{12} k_{11}^{\prime}-k_{22} k_{12}^{\prime}\right)+J_{\mu^{\prime}-\mu} J_{\mu-\mu^{\prime}-1} e^{+i \phi}\left(k_{11} k_{21}^{\prime}-k_{21} k_{22}^{\prime}\right)\right. \\
& \left.\left.+J_{\mu^{\prime}-\mu+1} J_{\mu-\mu^{\prime}} e^{-i \phi}\left(k_{11} k_{12}^{\prime}-k_{12} k_{22}^{\prime}\right)+J_{\mu^{\prime}-\mu-1} J_{\mu-\mu^{\prime}} e^{+i \phi}\left(k_{21} k_{11}^{\prime}-k_{22} k_{21}^{\prime}\right)\right]\right\} \\
& \left.+k_{\perp}^{2} T_{3} J_{\mu^{\prime}-\mu} J_{\mu-\mu^{\prime}}\left(k_{11} k_{11}^{\prime}+k_{22} k_{22}^{\prime}-k_{12} k_{21}^{\prime}-k_{21} k_{12}^{\prime}\right)\right) \text {, } \\
& \mathcal{S}_{12}=e^{-i\left(\mu-\mu^{\prime}\right) \phi} \frac{i}{\omega}\left(\left\{k _ { \| } T _ { 1 } \left(J_{\mu^{\prime}-\mu+1} J_{\mu-\mu^{\prime}-1} e^{+2 i \phi} k_{11} k_{22}^{\prime}+J_{\mu^{\prime}-\mu+1} J_{\mu-\mu^{\prime}+1} k_{12} k_{12}^{\prime}\right.\right.\right. \\
& \left.-J_{\mu^{\prime}-\mu-1} J_{\mu-\mu^{\prime}+1} e^{-2 i \phi} k_{22} k_{11}^{\prime}-J_{\mu^{\prime}-\mu-1} J_{\mu-\mu^{\prime}-1} k_{21} k_{21}^{\prime}\right) \\
& \left.\left.+k_{1} T_{2}\left[J_{\mu^{\prime}-\mu+1} J_{\mu-\mu^{\prime}} e^{+i \phi}\left(k_{11} k_{12}^{\prime}-k_{12} k_{22}^{\prime}\right)+J_{\mu^{\prime}-\mu-1} J_{\mu-\mu^{\prime}} e^{-i \phi}\left(k_{22} k_{21}^{\prime}-k_{21} k_{11}^{\prime}\right)\right]\right\}\right) \text {, } \\
& \mathcal{S}_{21}=e^{-i\left(\mu-\mu^{\prime}\right) \phi} \frac{1}{\omega}\left(\left\{i k _ { \| } T _ { 1 } \left(J_{\mu^{\prime}-\mu+1} J_{\mu-\mu^{\prime}+1} k_{12} k_{12}^{\prime}-J_{\mu^{\prime}-\mu+1} J_{\mu-\mu^{\prime}-1} e^{+2 i \phi} k_{11} k_{22}^{\prime}\right.\right.\right. \\
& \left.+J_{\mu^{\prime}-\mu-1} J_{\mu-\mu^{\prime}+1} e^{-2 i \phi} k_{22} k_{11}^{\prime}-J_{\mu^{\prime}-\mu-1} J_{\mu-\mu^{\prime}-1} k_{21} k_{21}^{\prime}\right) \\
& \left.\left.+k_{1} T_{2}\left[J_{\mu^{\prime}-\mu} J_{\mu-\mu^{\prime}+1} e^{-i \phi}\left(k_{12} k_{11}^{\prime}-k_{22}-k_{12}^{\prime}\right)+J_{\mu^{\prime}-\mu} J_{\mu-\mu^{\prime}-1} e^{+i \phi}\left(k_{21} k_{22}^{\prime}-k_{11} k_{21}^{\prime}\right)\right]\right\}\right) \text {, }
\end{aligned}
$$

$$
\begin{aligned}
\rho_{22}=e^{-i\left(\mu-\mu^{\prime}\right) \phi} T_{1}( & J_{\mu^{\prime}-\mu+1} J_{\mu-\mu^{\prime}-1} e^{+2 i \phi} k_{11} k_{22}^{\prime}-J_{\mu^{\prime}-\mu+1} J_{\mu-\mu^{\prime}+1} k_{12} k_{12}^{\prime} \\
& \left.+J_{\mu^{\prime}-\mu-1} J_{\mu-\mu^{\prime}+1} e^{-2 i \phi} k_{22} k_{11}^{\prime}-J_{\mu^{\prime}-\mu-1} J_{\mu-\mu^{\prime}-1} k_{21} k_{21}^{\prime}\right) .
\end{aligned}
$$


Before presenting numerical results and physical conclusions in the next section we briefly outline some mathematical and numerical aspects of the calculations.

(i) In Fig. 1 the graphic representation of the scattering amplitude shows that it implicitly contains the contribution of the vacuum polarization without a coupling to the external field. For different initial and final states this contribution is zero. However, numerically this representation of zero converges very slowly in terms of the sums and integrals in (34), and it is convenient to substract the free contribution in the reduced matrix elements:

$$
\mathcal{S}_{\lambda_{i} \lambda_{f}} \rightarrow \mathcal{S}_{\lambda_{i} \lambda_{f}}-\mathcal{S}_{\lambda_{i} \lambda_{f}}^{\text {free }} .
$$

(ii) In Sec. IV it was shown that the radial propagation is damped by a factor $\exp \left[-\left(\boldsymbol{M}+\boldsymbol{M}^{\prime}\right)\left|\rho_{2}-\rho_{1}\right|\right]$. Consequently the main contribution to the radial integrals comes from values of $p_{z}$ and $\zeta$ minimizing $\operatorname{Re}\left(M+M^{\prime}\right)$, $\left|\operatorname{Im}\left(\boldsymbol{M}+\boldsymbol{M}^{\prime}\right)\right|$.

(iii) For the incident angles under consideration, i.e., in the range from 1 to $10 \mu \mathrm{rad}$, the photon waves are directed nearly parallel to the $z$ axis and the sum over angular momenta converges well. From the explicit form of the $\mathcal{S}_{\lambda_{i} \lambda_{f}}$ it can be seen that the terms with small $\left|\mu-\mu^{\prime}\right|$ dominate the sums over angular momenta, where the terms with $\left|\mu-\mu^{\prime}\right|=1$ are about one order of magnitude bigger than the next ones, reflecting the spin-1 character of the photon.

(iv) The sum over "spins" $s, s^{\prime}$ can be done by means of Eq. (27).

(v) The contour $C_{F}$ should be rotated to the imaginary axis to improve the convergence of the $\zeta$ integration. This has to be done with care, since the cuts and poles of $\mathcal{f}$ have to be circumvented. For this purpose we remember the properties of $G\left(\mathbf{x}_{1}, \mathbf{x}_{2}, \zeta\right)$ as a function of complex energy $\zeta$. $G$ is a meromorphic function with branch cuts for real $\zeta,|\xi|>m_{\perp}$, and simple poles for $\zeta=E_{\alpha}$, with $E_{\alpha}$ the total energy of a bound state with quantum numbers $\alpha$. Since $G$ has real values for real $\zeta$, where defined, it obeys the reflection principle of Schwarz,

$$
G\left(\mathbf{x}_{1}, \mathbf{x}_{2}, \zeta^{*}\right)=G^{*}\left(\mathbf{x}_{1}, \mathbf{x}_{2}, \zeta\right)
$$

The reduced matrix element is given by products of the form

$$
G_{1}\left(\mathbf{x}_{1}, \mathbf{x}_{2}, p_{z}, \zeta\right) G_{2}\left(\mathbf{x}_{2}, \mathbf{x}_{1}, p_{z}^{\prime}=p_{z}+k_{\|}, \zeta^{\prime}=\zeta+\omega\right)
$$

and the cuts and poles of $\mathscr{S}$ are lying at the places sketched in Fig. 4. The lines represent the cuts and the crosses stand for the bound states. The thick lines give the new contour, split into five parts. The paths $C_{1}$ and $C_{5}$ give the contribution of the imaginary axis,

$$
\int_{C_{1}+C_{5}} \mathcal{S}(\zeta) d \zeta=\int_{0}^{i \infty}\left[\mathcal{S}(\zeta)-\mathscr{S}\left(\zeta^{*}\right)\right] d \zeta,
$$

where the integrand can be simplified by observing $k_{i j}^{*}(\zeta)=k_{i j}\left(\zeta^{*}\right)$. The integrals over $C_{2}$ and $C_{4}$ are closely

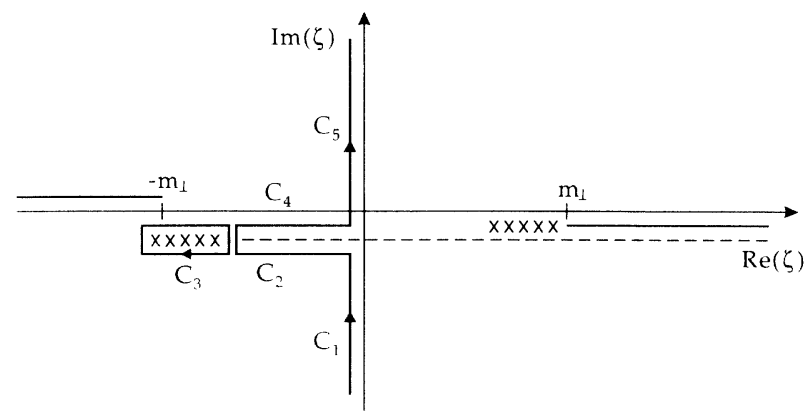

FIG. 4. Deformed contour of integration in the complex $\xi$ plane.

related too, and in a symbolic notation one gets

$$
\begin{aligned}
\int_{C_{2}+C_{4}} \mathcal{S}(\xi) d \xi & \sim \int_{C_{2}+C_{4}} G_{1}(\xi) G_{2}(\xi+\omega) d \xi \\
= & 2 \lim _{\delta \rightarrow 0} \int_{a}^{0} G_{1}(\xi-i \delta) \\
& \times \operatorname{Im}\left[G_{2}(\zeta+\omega+i \delta)\right] d \zeta,
\end{aligned}
$$

with $a=\min \left(0, m_{\perp}^{\prime}-\omega\right)$. The last contribution comes from the closed integral around the shifted poles of bound states. Using the form (20) of $G$ and Cauchy's theorem, the following expression remains:

$$
\begin{aligned}
\int_{C_{3}} \mathcal{S}(\zeta) d \zeta \sim-2 \pi i \sum_{\alpha^{\prime}} & k_{i j}\left(\rho_{1}, \rho_{2}, \zeta=E_{\alpha^{\prime}}-\omega, p_{z}\right) \\
& \times \widetilde{k}_{l m}\left(\rho_{2}, \rho_{1}, E_{\alpha^{\prime}}, p_{z}^{\prime}\right) .
\end{aligned}
$$

Here the sum is over all bound states in the primed quantities and $\widetilde{k}_{l m}\left(\rho_{2}, \rho_{1}, E_{\alpha^{\prime}}, p_{z}^{\prime}\right)$ is the radial propagator given by (25) with $\mathbf{v} \rightarrow \mathbf{u}$ and $\mathbf{w} \rightarrow \mathbf{u}, K\left(\zeta^{\prime}\right)=1$, where the normalized spinor $\mathbf{u}$ describes a bound state with quantum numbers $\alpha^{\prime}$, especially the total energy $E_{\alpha^{\prime}}$. Note that for the Doyle-Turner potential the sum in (47) involves only a finite number of bound states for given $\mu^{\prime}, p_{z}^{\prime}$. This is in contrast to a description in terms of a Coulomb-like potential, where the sum is infinite not only in $\mu^{\prime}$.

(iv) Since we are first of all interested in the order of magnitude of Delbrück scattering, we do not calculate $S_{f i}$ with high accuracy. The error in the absolute values of the results, however, should not be larger than $20 \%$. The accuracy could be improved by investing much more computer time, but our results prove already that photon channeling is exceedingly weak, such that it seems unreasonable to invest much more effort to improve accuracy.

Now we come to the results of the calculations. In Fig. 5 the squared $S$ matrix for an incident photon of $5 \mathrm{GeV}$ energy is shown. The incident angle is $\psi=1 \mu \mathrm{rad}$, and sum and average over initial and final polarizations is to be understood. Interestingly we have a periodic function of the "scattering angle" $\phi$ in the transverse momentum plane, and this shape is similar for all values of $\omega$ and $\psi$.

The periodicity of $|S|^{2}$ in $\phi$ is an interesting property, implying that the photon is preferably scattered in forward direction or is "bounced" by the string. 


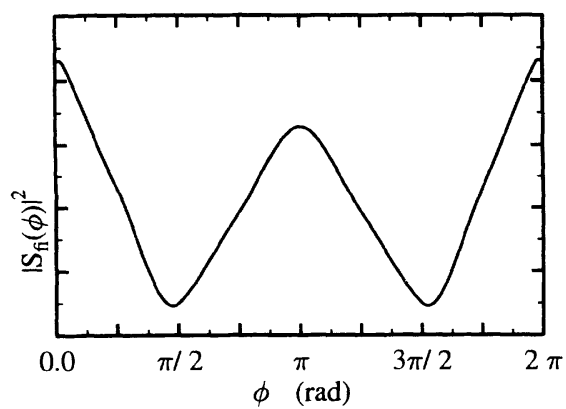

FIG. 5. Squared $S$ matrix in dependence of $\phi$; see text.

\section{AXIAL PHOTON CHANNELING}

With the $S$ matrix, calculated in the last section, we are able to evaluate a differential cross section per unit length of scattering the initial photon into a group of final states within the interval $[\phi, \phi+d \phi]$ :

$$
d \sigma=\frac{1}{\omega} \frac{\alpha^{2}}{64 \pi^{3}} \sum_{\lambda_{i}, \lambda_{f}}\left|S_{\lambda_{i} \lambda_{f}}(\phi)\right|^{2} d \phi
$$

To derive a probability, the cross section has to be divided by the area covered by one channel when "looking" at the front of the crystal $A_{C} \approx 7.6 \AA^{2}$.

Next we have to multiply with a typical length $L_{e}$. This is chosen to be

$$
L_{e}=D \frac{k_{\|}}{k_{\perp}},
$$

where $D \approx 0.2338 \AA$ is the transverse distance from the $z$ axis, where the potential has reached the value $V(0) / e$. Since the main contribution in the radial integrals comes from the radii of maximal electric field, $\rho \approx 0.125 \AA$, this seems to be a reasonable estimate. Finally we multiply the derived probability with $\phi$ and integrate over the range of angles. The result is an average angle of rotation for the transverse photon momentum

$$
\Phi=\frac{D}{A_{C}} \frac{\alpha^{2}}{64 \pi^{3}} \frac{k_{\|}}{k_{\perp} \omega} \int_{0}^{2 \pi} \sum_{\lambda_{i}, \lambda_{f}}\left|S_{\lambda_{i} \lambda_{f}}(\phi)\right|^{2} \phi d \phi .
$$

With this formula we derived rotation angles for various photon energies and incident angles. Before presenting the numerical results we first want to give an intuitive description of our criteria for the occurrence of photon channeling.

The important question for us is whether the photon stays close to one string, i.e., in the string field, rather than how large an angle it is scattered during its passage through the target crystal. The latter would get contributions from independent scatterings at different strings. To be bound at one string the photon should at least reverse its momentum before it could leave the string potential. Therefore photon channeling cannot take place if $\Phi \ll 2 \pi$. On the other hand, channeling becomes possible if $\Phi$ is becoming large, $\Phi \gg 2 \pi$. One should not take this picture too literally, because in the continuum description we have neglected the $z$ dependence of the po-

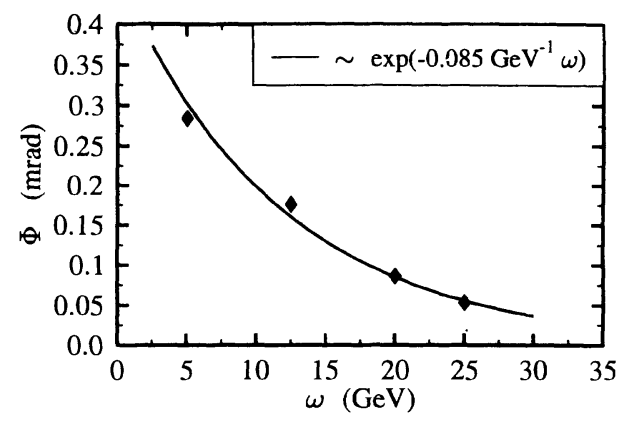

FIG. 6. Dependence of $\Phi$ on photon energy $\omega$; incident angle $\psi=1 \mu \mathrm{rad}$.

tential. In a real crystal the photon can change $k_{\|}$as well as $k_{1}$, not only the direction of $k_{\perp}$ in the transverse plane. However, our results should give the correct order of magnitude and thus should allow one to decide whether a photon can be channeled.

In Fig. 6 the dependence of $\Phi$ on the photon energy is shown for an incident angle of $\psi=1 \mu \mathrm{rad}$. The diamonds mark the results of the calculation and the curve is a fit to the data points, showing that $\Phi$ is softly decreasing with energy $\omega$. The absolute values of $\Phi$ are lying in the region of mrad, implying that no channeling effect for these photons can be expected. At this point a short remark on the Compton scattering of the photons should be made. A simple estimate, multiplying the total Compton cross sections for one electron with the number of target atoms per length $L_{e}$ and with $Z_{G e}=32$, shows that for the energies under consideration Compton scattering can be neglected.

Figure 7 shows $\Phi$ as a function of the incident angle $\psi$ for the energy $\omega=5 \mathrm{GeV}$. The data points are fitted very well by a line proportional to $\psi^{-1}$. This dependence means that only photons with very small incident angles $\psi<<1 \mu \mathrm{rad}$ could be channeled. These results can be applied to bremsstrahlung photons that are emitted by axially channeled electrons. In Fig. 8 the distribution in $\psi$ of these photons for an incident electron of $50 \mathrm{GeV}$ is shown, calculated within the SSM [16]. It is determined after a passage of $1 \mathrm{~mm}$ through the crystal, and the number of photons are summed up for three intervals of energy $\omega$. As expected, the distribution in $\psi$ becomes narrower for higher photon energies. The most interesting detail in our context is that for angles $\psi \leq 25 \mu \mathrm{rad}$ no

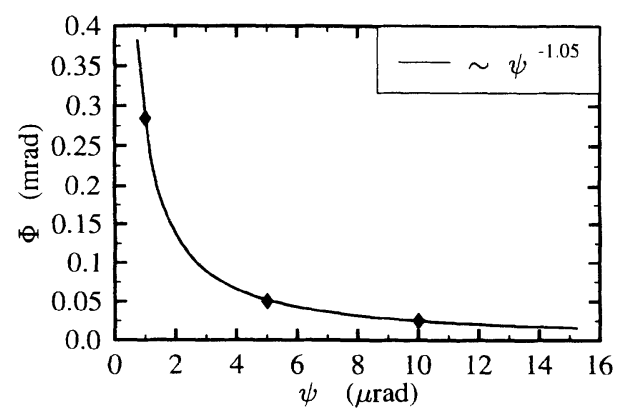

FIG. 7. $\Phi$ as a function of incident angle $\psi$, with $\omega=5 \mathrm{GeV}$. 


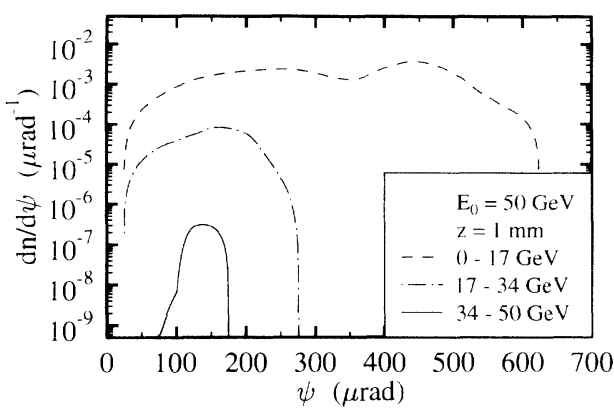

FIG. 8. Angular distribution for bremsstrahlung photons of an electron with initial energy of $50 \mathrm{GeV}$; see text.

significant number of photons is emitted. Remembering the results for $\boldsymbol{\Phi}=\boldsymbol{\Phi}(\omega, \psi)$ we conclude that no channeling effect for bremsstrahlung photons can be expected.

Our results imply that photons are not channeled for attainable energies. $\Phi$ does not increase strongly with energy, showing that the nonperturbative QED effects encountered in channeling are qualitatively different from those in, e.g., strong Coulomb fields [17,18]. Pair creation in channeling is then no sign of overcriticality. To elucidate this point let us analyze the electromagnetic field experienced by an electron with half the photon energy (see Fig. 9).

Obviously both the electric and magnetic field grow with the Lorentz factor $\gamma_{e}$ of the electron. [For $\omega=25$ $\mathrm{GeV}$ one has, e.g., $\gamma_{e}=\left(\begin{array}{lll}12.5 & \mathrm{GeV}\end{array}\right) /(0.5 \mathrm{MeV})$ $\approx 2.5 \times 10^{4}$.] Thus the electromagnetic interaction becomes completely nonperturbative and the electric fields alone become overcritical. However, simultaneously the magnetic field, which is proportional to $\gamma_{e}$ too, reduces the radial mobility of electrons and positrons. Their "Landau orbit" has a width of $\sim 1 / B$. Both effects seem to cancel numerically. With this interpretation in mind and based on our numerical results we conclude that photon channeling does not occur for any reasonable energy. We do not believe that any improvement of the calculation, e.g., by taking the periodic oscillations in the $z$ direction into account, can change this conclusion.

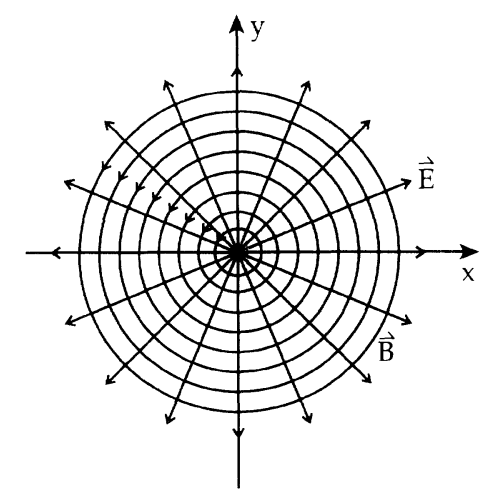

FIG. 9. Electromagnetic fields affecting an electron; see text.

\section{SUMMARY}

We have derived the exact electron propagator for axial channeling. This propagator can be used to calculate any QED process for the channeling situation; e.g., radiative corrections, photon splitting, etc. We used this propagator to calculate the interaction of a high-energy photon with the collective electromagnetic field of an oriented single crystal. We give numerical results for the average scattering angle as a function of incident angle and photon energy up to $25 \mathrm{GeV}$. We find that photons can indeed be channeled if they are sufficiently close to a channeling axis and their transverse momentum with respect to this axis is sufficiently small. The photons produced during high-energy electron channeling have, however, rather large transverse momentum and are produced relatively far from the channeling axes such that no appreciable channeling should take place.

\section{ACKNOWLEDGMENTS}

This work was supported by the Deutsche Forschungsgemeinschaft (G. Hess program). We thank J. Reinhardt, A. Scherdin, G. Soff, and A. Solovyov for helpful discussions.
[1] P. Rullhusen, F. Smend, M. Schumacher, A. Hanser, and H. Rebel, Z. Phys. A 293, 287 (1979); P. Rullhusen, U. Zurmühl, F. Smend, M. Schumacher, H. G. Börner, and S. A. Kerr, Phys. Rev. C 27, 559 (1983).

[2] A. Schäfer, J. Phys. G 15, 373 (1989).

[3] A. Schäfer, S. Graf, J. Augustin, W. Greiner, and E. Uggerhøj, J. Phys. G 16, L131 (1990).

[4] J. Lindhard, Mat. Fys. Medd. Dan. Vid. Selsk. 34, No. 14 (1965).

[5] A. H. Sørensen and E. Uggerhøj, Nuclear Science Applications (Harwood Academic, Chur, 1989), Vol. 3, p. 147.

[6] J. C. Kimball and N. Cue, Phys. Rep. 125, 69 (1985).

[7] D. S. Gemmel, Rev. Mod. Phys. 46, 129 (1974).

[8] P. A. Doyle and P. S. Turner, Acta Crystallogr. Sec. A 24, 390 (1968).

[9] S. P. Møller, E. Uggerhøj, H. W. Atherton, M. Clément, N. Doble, K. Elsener, L. Gatignon, P. Grafström, M.
Hage-Ali, and P. Siffert, Phys. Lett. B 256, 91 (1991).

[10] J. Klenner, Diploma thesis, University of Frankfurt am Main, 1991.

[11] G. V. Shishkin and V. M. Villalba, J. Math. Phys. 30, 2132 (1989); 30, 2373 (1989).

[12] V. G. Bagrov and D. M. Gitman, Exact Solutions of Relativistic Wave Equations (Kluwer, Dordrecht, 1990).

[13] C. Itzykson and J.-B. Zuber, Quantum Field Theory (McGraw-Hill, New York, 1980).

[14] G. E. Brown and G. W. Schaefer, Proc. R. Soc. London, Ser. 233, 527 (1956).

[15] E. H. Wichmann and N. M. Kroll, Phys. Rev. 101, 843 (1956).

[16] J. Augustin (unpublished).

[17] G. Bassompierre et al., Europhys. Lett. 22, 239 (1993).

[18] W. Greiner, B. Müller, and J. Rafelski, Quantum Electrodynamics of Strong Fields (Springer, Berlin, 1985). 